\title{
Myeloid sarcoma as a differential diagnosis of small bowel obstruction
}

\author{
Bastian Kettler ${ }^{1^{*}}$, Mahmoud Abbas ${ }^{2}$, Bastian Ringe ${ }^{1}$, Lothar Hambach ${ }^{3}$, Jürgen Klempnauer ${ }^{1}$, \\ Michael Winkler ${ }^{1}$, Hans-Heinrich Kreipe ${ }^{2}$, Nikos Emmanouilidis ${ }^{1}$ \\ ${ }^{1}$ Clinic for General-, Abdominal- and Transplant-Surgery, Medical School Hannover, Hannover, Germany; \\ *Corresponding Author: kettler.bastian@mh.hannover.de \\ ${ }^{2}$ Institute for Pathology, Medical School Hannover, Hannover, Germany \\ ${ }^{3}$ Clinic for Haematology, Medical School Hannover, Hannover, Germany
}

Received 24 December 2012; revised 1 April 2013; accepted 16 April 2013

Copyright (C) 2013 Bastian Kettler et al. This is an open access article distributed under the Creative Commons Attribution License, which permits unrestricted use, distribution, and reproduction in any medium, provided the original work is properly cited.

\begin{abstract}
We are presenting the case of a 42-year-old male patient, who was hospitalized due to an acute small bowel obstruction caused by a tissue mass of the mesentery. The patient reported that he had a history of a testicular tumour. For therapy of intestinal obstruction as well as for diagnostic reasons we decided to perform an explorative laparotomy. On histopathological examination the immunohistological staining was positive for myeloperoxidase (MPO) and KP-1 (CD68). Staining was slightly positive for Bcl-2, CD117, CD34, but negative for CD3, CD4, CD5, CD8, CD20, CD30, CD79, Bcl-6 and S-100. This leads to the diagnosis of a myeloid sarcoma. After recovery from surgery and chemotherapy, allogenic bone marrow transplantation was performed. Most intestinal obstructions are caused by postoperative adhesions or hernias and only in rare cases caused by a myeloid sarcoma.
\end{abstract}

Keywords: Myeloid Sarcoma; Small Bowel Obstruction

\section{INTRODUCTION}

An extramedullary myeloid sarcoma is a very rare tumour entity with an incidence of 2 in 1,000,000 [1]. Analogue to its greenish colour these types of myeloid sarcoma are named chloroma (gr. chloros = green) [2]. A chloroma might be found in any organ, but in the digestive tract, the bowl is the predominant site of manifestation [3].

\section{MATERIAL AND METHODS}

- The histopathological examination was done by Abbas M. (Junior Pathologist) and Kreipe H.H. (Senior Professor of Pathology).

- From each block, five sets of stained slides were produced, including slides stained with hematoxylineosin stain and an unstained section for individual use.

- The control pictures are done without the primary antibody

- Sections $4 \mu \mathrm{m}$ thick were prepared on APES-coated slides. After routine deparaffinisation and rehydration the sections were boiled in an EDTA citrate buffer in a pressure cooker for $2 \mathrm{~min}$ for antigen retrieval. The immunostains were performed using the avidin-biotin peroxidase complex amplification system in an automated immunostainer (NexES; Ventana Medical Systems, Tucson, Arizona, USA). Primary antibodies were obtained from Dako (Glostrup, Denmark) or Ventana Medical Systems.

- The RT-PCR analysis of the bone marrow was done after a standardised protocol, the bone marrow cells were enriched by Ficoll-Hypaque density gradient centrifugation. Quantification of CBFB-MYH11 fusion transcript expression for the three major fusion subtypes $\mathrm{A}, \mathrm{D}$, and $\mathrm{E}$ was performed by RQ-PCR using an ABI PRISM 7700 Sequence Detection System (Applied Biosystems, Darmstadt, Germany). Primers and probes were used according to the Europe Against Cancer standard protocols with GAPDH as housekeeping gene [5].

\section{CASE REPORT}

A 42-year-old male patient was hospitalized due to 
acute abdominal pain. The patient reported that he had a history of a testicular tumour with synchronous lymph node metastasis at the age of $26 \mathrm{yrs}$, which was treated by surgery and chemotherapy. Eleven years later he was diagnosed with a second non-seminoma tumour of the other testicle, which was removed as well. The patient was followed-up continuously by his outpatient urologist.

A computer tomography (CT) on admission did show an intestinal obstruction caused by a tumorous mass of the mesentery (Figure 1). For therapy of intestinal obstruction as well as for diagnostic reasons we decided to perform an explorative laparotomy. At surgery we found a massive tumour located in the middle of the small bowl's mesentery, as well as a smaller satellite tumour. The satellite tumour was the main reason for the obliteration of intestinal passage. A debulking of the tumorous mass of the mesentery as well as a small bowl short segment resection with complete removal of the satellite tumour was performed.

On the histo-pathological examination the tumour presented with blasty form like cells (Figure 2). The immunohistological staining was positive for KP-1 (CD68) and myeloperoxidase (MPO) (Figures 3 and $\mathbf{4}$ ). The control pictures (without the primary antibody) are given at Figures 5 and 6; further pictures are in the acknowledgments. The MIB -1 (Ki67) index was 30\% positive (not shown). Staining was slightly positive for Bcl-2, CD117, CD34, but negative for CD3, CD4, CD5, CD8, CD20, CD30, CD79, Bcl-6 and S-100 (not shown). The bone marrow biopsy was negative for acute leukaemia, but the genetic analysis with RT-PCR did show an inversion of chromosome 16 [5].

The patient quickly recovered from surgery and after induction chemotherapy with Danorubicin and Cyatarabin, allogenic bone marrow transplantation was per-

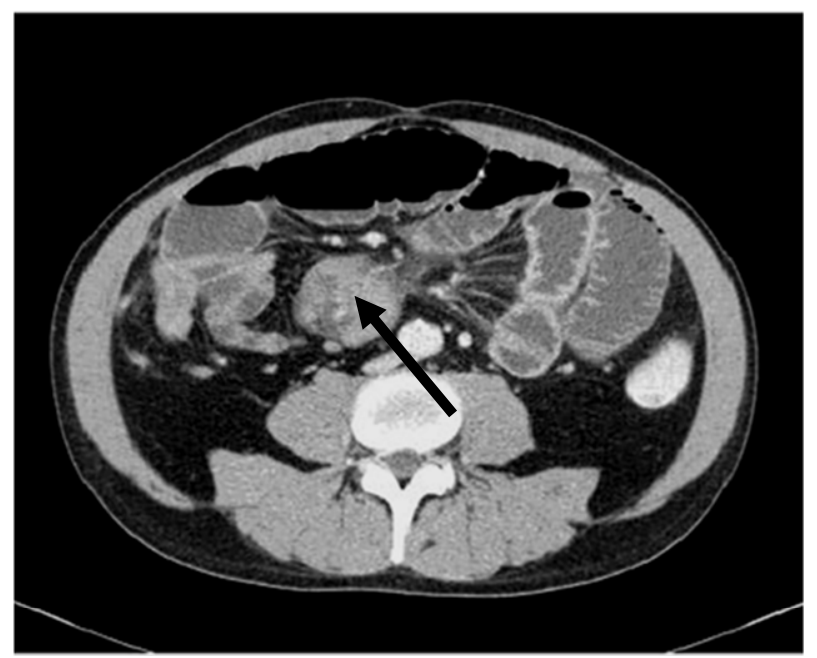

Figure 1. CT Abdomen 1. Arrow: abdominell mass.

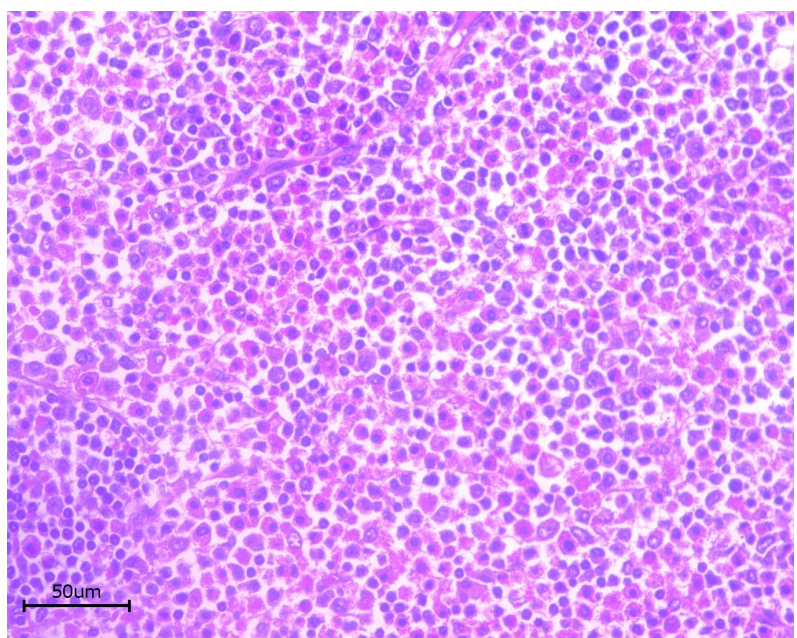

Figure 2. Myeloid sarcoma (Staining: HE).

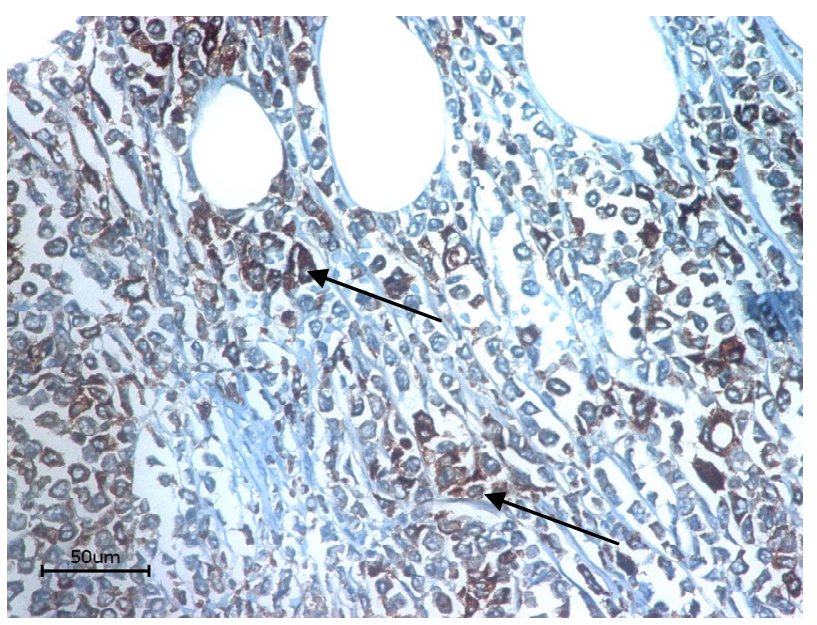

Figure 3. KP1 (CD 68). Arrows: positive cells.

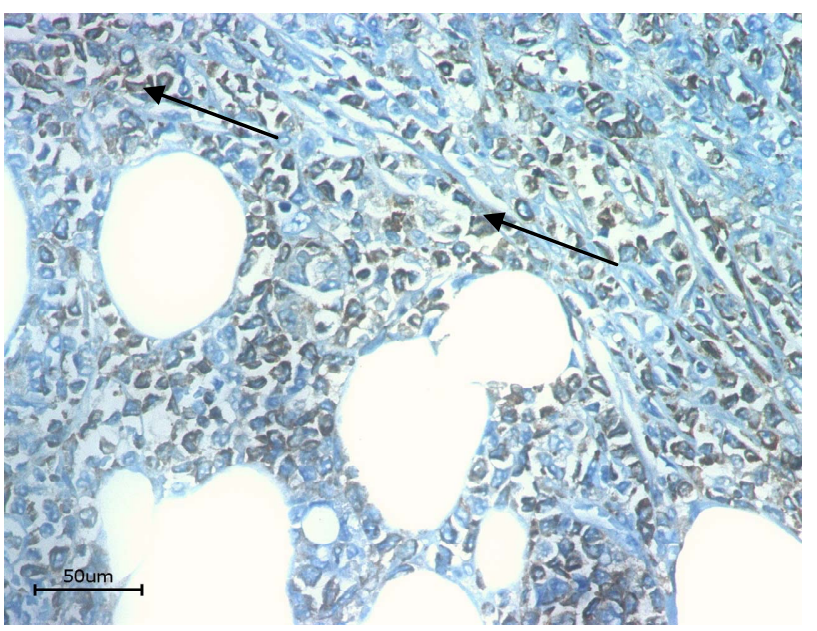

Figure 4. Myeloperoxidase. Arrows: positive cells.

formed. One year after surgery and 6 month after bone marrow transplantation, the patient is remission free and in a good clinical condition. 


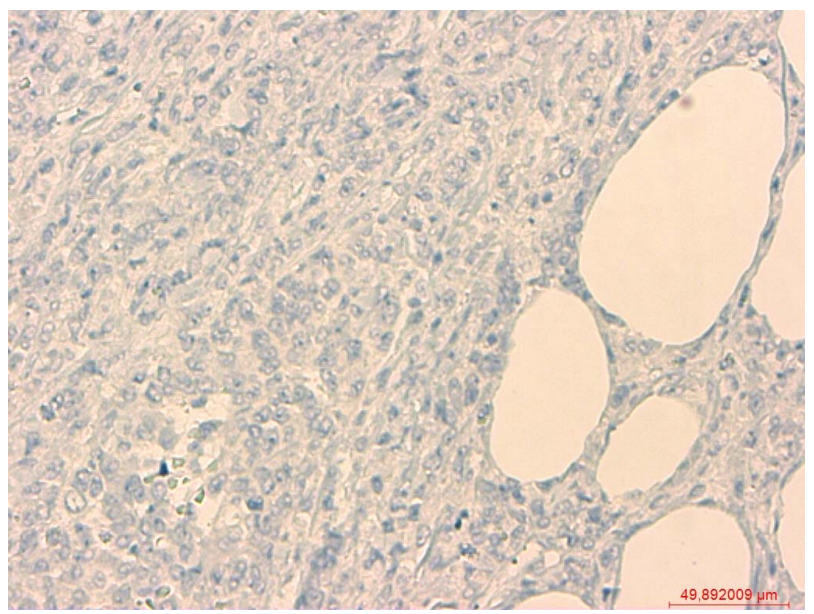

Figure 5. Control staining (KP1/CD68).

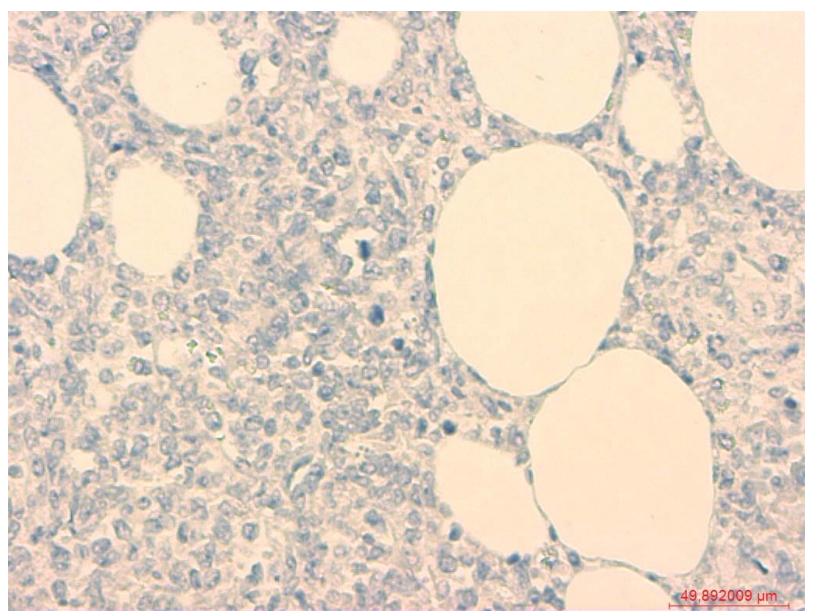

Figure 6. Control staining (MPO).

\section{DISCUSSION}

Postoperative adhesions and hernias are the main reasons for obliteration of intestinal passage, and only in very few occasions tumours of the small bowl have been reported as reasons for intestinal obstruction [6]. Even fewer of those where related to a myeloid sarcoma associated tumour mass $[7,8]$.

The primary diagnosis of a myeloid sarcoma is very difficult, because there are no specific signs or symptoms [3]. The diagnosis is based on the pathologic results; however misdiagnoses are not uncommon $[1,4]$. In this case with the history of a testicular cancer and chemotherapy, a secondary neoplasm had to be taken into account. The resection of the intestine has been necessary in this case, because of the mechanic compression of the small intestine. In general, these kinds of diseases cannot be cured with an operation. A systematic therapy including chemotherapy and bone marrow transplantation is necessary. Due to its rarity and the lack of data the few known cases of a myeloid sarcoma in the intestine, the

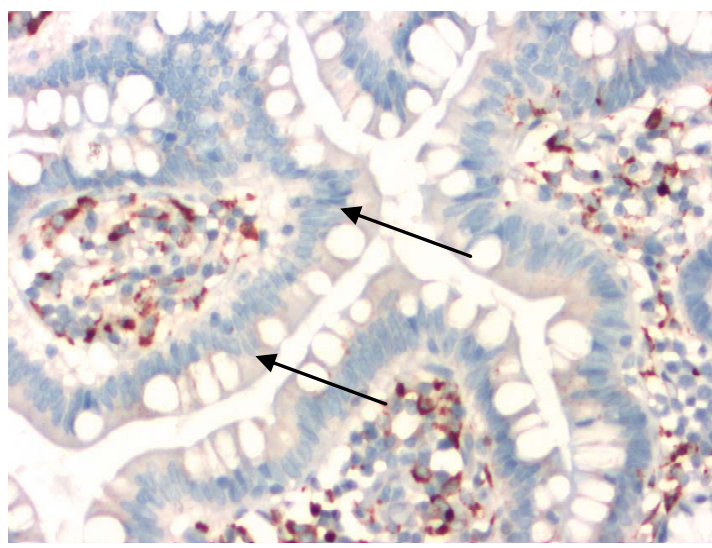

Figure 7. KP 1 (CD68).

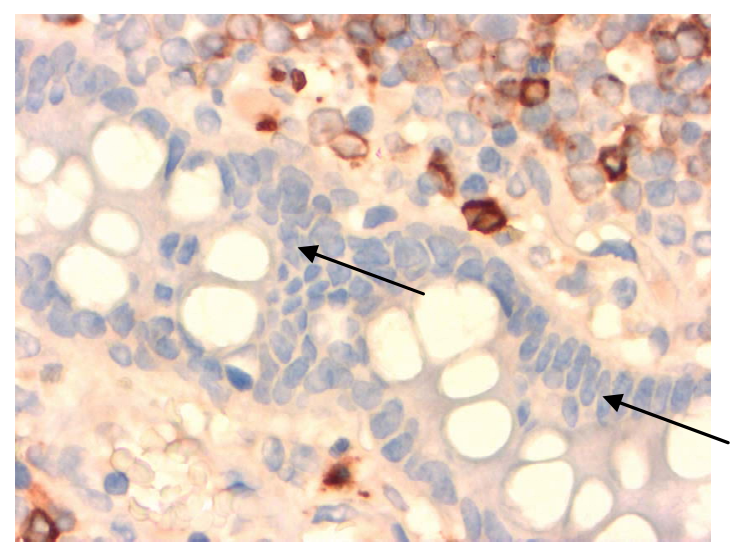

Figure 8. MPO.

therapy follows protocols for acute myeloid leukaemia $[3,9]$.

\section{ACKNOWLEDGEMENTS}

- Figure 2 is a hematoxylin eosin stained picture, which represents an overview of the blasty form mass of cells.

- Figure $\mathbf{3}$ and $\mathbf{4}$ are stained with the monoclonal (primary) antibodies CD68/ myeloperoxidase and the secondary antibody DAB (3,3' Diaminobenzidin) and HRP (horseradish peroxidase). The molecules CD68 and myeloidperosidase are both localized in the cytoplasm of the cell. Because of using DAB and HRP the substrate (after transgression of the solubility product) is visible as a brown color. The arrows in the pictures 3 and 4 show these brown deposits.

- The Figure 5 and 6 are the control pictures of 3 and 4, which were stained without the primary antibody.

- Figures $\mathbf{7}$ and 8 are staining with the antibodies against KP1 (CD68) (Figure 7) and MPO (Figure 8). In both cases, negative epithelia cells of the small intestine are highlighted by arrows.

\section{REFERENCES}

[1] Antic, D., Elezovic, I., Bogdanovic, A., Vukovic, N.S., Pavlovic, A., Jovanovic, M.P., et al. (2010) Isolated mye- 
loid sarcoma of the gastrointestinal tract. Internal Medicine Journal, 49, 853-856.

doi:10.2169/internalmedicine.49.2874

[2] Sivan-Hoffmann, R., Waksman, I., Cohen, H.I. and Eitan, A. (2011) Small bowel obstruction as a presenting sign of granulocytic sarcoma. The Israel Medical Association Journal, 13, 507-509.

[3] Kohl, S.K. and Aoun, P. (2006) Granulocytic sarcoma of the small intestine. Archives of Pathology \& Laboratory Medicine, 130, 1570-1574.

[4] Kumar, B., Bommana, V., Irani, F., Kasmani, R., Mian, A. and Mahajan, K. (2009) An uncommon case of small bowel obstruction: Isolated primary granulocytic sarcoma. QJM: An International Journal of Medicine, 102, 491493. doi:10.1093/qjmed/hcp051

[5] Corbacioglu, A., Scholl, C., Schlenk, R.F., Eiwen, K., Du, J., Bullinger, L., Fröhling, S., et al. (2010) Prognostic Impact of Minimal Residual Disease in CBFB-MYH11Positive Acute Myeloid Leukemia. Journal of Clinical Oncology, 28, 3724-3729.

\section{doi:10.1200/JCO.2010.28.6468}

[6] Sarraf-Yazdi, S. and Shapiro, M.L. (2010) Small bowel obstruction: The eternal dilemma of when to intervene. Scandinavian Journal of Surgery, 99, 78-80.

[7] Yamauchi, K. and Yasuda, M. (2002) Comparison in treatments of nonleukemic granulocytic sarcoma. Report of two cases and review of 72 cases in the literature. American Cancer Society, 94, 1739-1746.

[8] Tsimberidou, A.M., Kantarjian, H.M., Estey, E., Cortes, J.E., Verstovsek, S., Faderl, S., et al. (2003) Outcome in patients with nonleukemic granulocytic sarcoma treated with chemotherapy with or without radiotherapy. Leukemia, 17, 1100-1103. doi:10.1038/sj.leu.2402958

[9] Tsimberidou, A.M., Katarjian, H.M., Wen, S., Keating, M.J., Susan, B., Brandt, M., Pierce, S., Freireich, E.J., Mederiros, J.M. and Estey, E. (2008) Myeloid sarcoma is associated with superior event-free survival and overall survival compared with acute myeloid leukemia. Cancer, 113, 1370-1378. 